\title{
Real-Time Multi-View SAR Imaging Using a Portable Microwave Camera with Arbitrary Movement
}

\author{
Jaime Laviada, Mohammad Tayeb Ghasr, Senior Member, IEEE, Miguel López-Portugués, Fernando Las-Heras, \\ Senior Member, IEEE, and Reza Zoughi, Fellow, IEEE
}

\begin{abstract}
This paper presents the first demonstrator of a portable, multi-view, high-resolution, three-dimensional (3D) and real-time microwave imaging system. The system is based on a recently-developed real-time 3D microwave camera, which performs quasi-monostatic acquisitions, equipped with an optical depth camera providing target surface profile information. Additionally, the entire system can be arbitrarily moved along the target performing microwave and depth camera synchronized acquisitions from different views with a twofold purpose, namely; a) enabling a coverage area much larger than that possible with a static imaging system, and b) allowing for incorporation of several tilt angles (or views) to enhance capturing specular reflection imaging data to improve the overall image quality. At each scanning position, the imaging data from the microwave camera are processed to build a local 3D microwave image. The information is then merged, using recently-proposed techniques for multi-view synthetic aperture imaging, to compose the global image. The synchronized optical camera depth acquisitions enable tracking the entire imager movements so that the position and attitude are known. Moreover, the data acquired by the depth camera are also use to build a complementary 3D outer surface profile model of the target, producing a combined and realistic image of the internal and external geometries of the target. Finally, the performance of the combined system is evaluated using several examples related to hidden contraband covered by clothing (i.e., people screening).
\end{abstract}

Index Terms-3D microwave camera, multi-view imaging, synthetic aperture imaging, depth camera.

\section{INTRODUCTION}

M ICROWAVE (and millimeter wave) imaging is a powerful tool for evaluating the internal composition and structures of microwave penetrable targets. A large variety of imaging system configurations and post-processing techniques have been developed to carry out this type of imaging depending on the nature of the problem and the available resources [1]. Noteworthy among the many viable and practical applications of these techniques are nondestructive evaluation [2], [3], screening of people [4] and medical imaging [5], [6].

Some of the most effective approaches in this area are based on synthetic aperture radar (SAR) imaging techniques

This work has been partially supported by the Ministerio de Ciencia e Innovación of Spain /FEDER under project TEC2014-55290-JIN.

Jaime Laviada, Miguel López-Portugués and Fernando Las-Heras are with the Department of Electrical Engineering, University of Oviedo, Gijón, 33203, Spain (e-mail: jlaviada@tsc.uniovi.es; mlopez@tsc.uniovi.es; flasheras@uniovi.es).

Mohammad Tayeb Ghasr and Reza Zoughi are with the Applied Microwave Nondestructive Testing Laboratory, Missouri University of Science and Technology, Rolla, MO 65409 USA. (e-mail: mtg7w6@mst.edu; zoughi@mst.edu). in which the scattered field is acquired along a 2D plane, usually referred to as (measurement or imaging) aperture [7], [8], [9]. Depending on the arrangement between the transmitters and receivers, a monostatic [4], [7] or multi-static [10] measurement approach can be implemented. However, unless a voluminous measurement system, consisting of a large aperture, is implemented to physically encompass a relatively large object, such as a human body (e.g., today's airport millimeter wave screening booths), only local areas of the object under test can be imaged. To increase the observation area, measurement schemes combining several fixed apertures have been proposed [11], [12] in order to generate a collection of independent images from different positions. In addition, for people screening applications, some methods incorporate the natural movement of a human being, enabling complementary data acquisition [13]. Moreover, if the imaging system (i.e., aperture) does not encompass the entire body under test, then imaging data from specular direction may not be collected, and some areas of the body under test may not be imaged [14].

Using conventional optical cameras, multiple view information, obtained from several arbitrary positions, is often employed for several purposes. For example, this information has been used to estimate the attitude and position of a camera as well as building a 3D model from bi-dimensional pictures [15]. This has resulted in the implementation of 3D (optical) scanners, which can be arbitrarily moved [16]. In contrast, SAR imaging is often implemented from a single aperture (synthetic or real) position, referred to as a view (analogous to computer vision terminology). These current capabilities of conventional optical cameras, coupled with the recent development of portable wideband (3D) microwave cameras [17], has been the impetus for this work, namely; the production of multi-view electromagnetic images. The latter, used in this work, consists of a $20-30 \mathrm{GHz}$ real-time, portable microwave camera with an aperture of $\sim 250 \mathrm{~mm} \times 230 \mathrm{~mm}$ capable of producing 3D images of objects [17]. As we will demonstrate in this paper, combining the imaging data from both of these cameras enables image production of objects much larger than the microwave camera aperture alone. In a similar fashion to conventional optical cameras, in this work microwave data is acquired from different positions resulting from arbitrary movements. The feasibility of this novel concept was initially demonstrated in [18] by merging optical cameras and SAR imaging resulting from moving/scanning a single 
transceiver to synthesize the aperture. Furthermore, it was demonstrated that the proposed algorithms could also yield 3D object surface profile, obtained from the optical camera, and images of the interior of the object, obtained from the electromagnetic imaging data. In addition, the capability of using different tilted views for the same position provides a valuable mechanism to overcome the aforementioned drawback regarding specular reflections when using relatively small imaging apertures [14].

Further research to extend [18] was focused on sparse data collection to reduce the number of antenna elements for the multi-view imager [19] as well as on replacing the conventional optical camera by one capable of producing object depth information [20], which can provide not only the RGB values of each pixel but also its depth. This is important since this also improves pixel position accuracy for texture-less objects (e.g., plain clothing). However, all of the aforementioned works were conducted and validated by raster scanning a single antenna and then performing imaging data post-processing offline. In contrast, in this work, a fully functional and portable multi-view microwave camera capable of producing 3D real-time images is used. This imager (i.e., microwave and depth cameras), comprising the microwave camera presented in [17] and a depth camera, is portable and it retains the capability for building the double model on-the-fly as the operator moves the system along arbitrary trajectories.

This work technically extends the preliminary work presented in [21], in which the positioning system was not included but the microwave camera was placed at known positions and the imaging data was then processed offline. Here, we first summarize the hardware components, such as the microwave and the depth cameras, as pertinent to the objectives of this work, including the positioning system based on the latter. Subsequently, data stream processing from both cameras is presented via a flowchart detailing the relevant steps and the need for synchronization between the two data streams. Finally, several examples, related to concealed weapon screening, are illustrated showing the efficacy of the scanner for such critical applications.

\section{Multiview PORTABle IMAGER}

The main goal of the approach described here is to build a three-dimensional model of a body under test with a real-time, portable and 3D microwave camera [17]. The combination of this microwave camera and a depth camera results in an imager that can dynamically acquire both data sets and produce a $3 \mathrm{D}$ image on an object of interest as the two are moved around the object. For this purpose, the multi-view paradigm from conventional optical cameras [22] is employed. In the case of microwave camera, the ability to obtain multiple views of an object is of critical importance for two key reasons. First, portable cameras are expected to be relatively small in size. Consequently, they cannot build a complete image of a larger object under test (see Fig. 1). Second, due to the relatively small imaging aperture size, there is a high likelihood of missing the scattered signal due to specular reflections [14]. To remedy this, tilting and moving the imaging aperture will be needed to produce a representative image of the object, as depicted in Fig. 2.

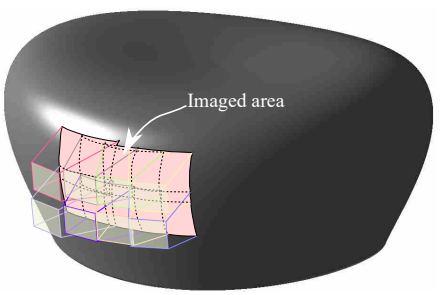

(a)

(b)

Fig. 1. Multi-view scanning: (a) imaged area with a single-view scheme, and (b) imaged area with a multi-view scheme.

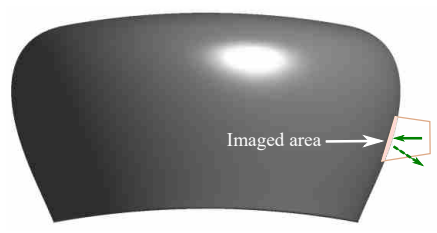

(a)

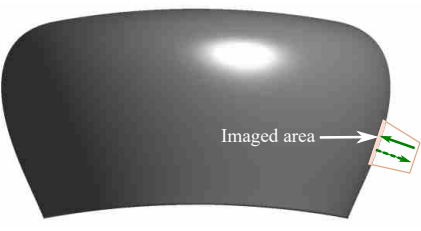

(b)
Fig. 2. Possible reflections in case of a single-view system: (a) oblique incidence, and (b) normal incidence.

To build such an imaging system as proposed three main system components are required, namely: $i$ ) microwave data acquisition; ii) positioning system and; iii) control and data processing, as described below.

\section{A. Microwave data acquisition}

The cornerstone of the portable scanner is the portable microwave camera (one-sided or monostatic) with the capability of providing sufficient amount of wideband data to produce a 3D local image of a portion of a large object in real-time [17]. Although other cameras with different architectures are also available (e.g., [23]), the use of a monostatic architecture is preferred as it does not require and an external illumination source, which among other attributes helps to keep the overall system compact.

A detailed description of this microwave camera is provided in [17] and, therefore, only the main characteristics and features relevant to the presented work are summarized here. The camera is made of 16 interlaced boards, each of them containing 16 equally-spaced tapered slot antennas with two built-in receivers. Thus, the overall number of spatial data acquisition points is $16 \times 32$. Although the receivers are equally-spaced along the same boards, there is a shift of $4 \mathrm{~mm}$ between the boards. Consequently, the acquisition points for the quasi-monostatic system are placed at a nonregular lattice. The overall dimensions of the camera are $\sim 260 \times 210 \times 180 \mathrm{~mm}$ and its weight is $4.8 \mathrm{~kg}$. The feeding network consists of several cascaded amplifiers, multiplexers and switches fed by a single voltage controlled oscillator (VCO), which continuously sweeps from $20 \mathrm{GHz}$ to $30 \mathrm{GHz}$. The power at each antenna input is $\sim 10 \mathrm{dBm}$ (see [17] for a complete power balance of the feeding network). The VCO 
sweep time is set so that the camera can provide wideband data at an equivalent rate of 30 frames per second. The built-in receivers provide a baseband signal sampled by a two-channel ADC, whose data are collected and stored by a Beaglebone Black board. This board also provides connectivity with an external computer via an Ethernet connection.

Although the camera has been used to detect targets up to standoff distances of $\sim 30 \mathrm{~cm}$, its best performance is achieved at less than $10 \mathrm{~cm}$ since not only the signal-to-noise ratio decreases due to the propagation losses but also the spatial resolution of aperture-limited SAR imagers degrades as a function of increasing standoff distance [4]

\section{B. Positioning system}

The portable scanner must be equipped with a subsystem capable of providing position and attitude of the camera so that the electromagnetic data from multiple views can be properly merged. As discussed in the previous section, the microwave camera can perform a full acquisition in approximately $33 \mathrm{~ms}$, and therefore, movement is not expected to have a significant impact in a locally-obtained image. Nevertheless, the displacement distance between multiple views can be electrically large since the processing time, although fast, is non-negligible. The optimal choice for a positioning system is then one that can provide position information with an error much smaller than the minimum operating wavelength, corresponding to $30 \mathrm{GHz}$. If this condition for the positioning error is satisfied, then the images can be coherently merged [14].

At the considered frequencies, the required small positioning error can be achieved using laser trackers or optical systems based on markers. Nonetheless, these methods were not considered here since their implementation requires the use of external equipment in the line-of-sight of the imager. Other positioning schemes are based on photogrammetry. These systems, which require a conventional inexpensive optical camera, cannot estimate the position with an error significantly smaller than the microwave wavelength [18]. Consequently, an incoherent merging scheme must be employed. For those merging schemes, positioning inaccuracies result in artifacts whose sizes are similar to the positioning error [14]. If the object under test is rich in textures, so that key points on its surface can be identified, then a positioning error in the order of a few millimeters results, which is tolerable for these suboptimum systems.

In this work, the positioning scheme is based on a depth camera following the procedure outlined in [20]. These cameras have an advantage over the conventional optical cameras as they provide a 3D point cloud image instead of bidimensional one. The position and attitude (i.e., roll, pitch and yaw angles) of the camera can be found by comparing consecutive point clouds without the need to resort to color information. This makes the approach robust in cases in which the area under test does not have any relevant edges or flat textures by which to detect required key points as well as independent of variations in lighting conditions [18]. In the presented system, a low-cost Intel® RealSense ${ }^{\text {TM }}$ SR300 depth camera [24] is used. This camera is based on structured light [25] and it provides three-dimensional point clouds of dimensions $640 \times 480$ at a framerate of 60 frames per second.

\section{Data processing}

Wideband synthetic aperture radar techniques build images from the reflectivity function, which measures the fraction of the incident signal reflected by each point in a scene [26]. This 3D function can be represented by different approaches (single cut [14], stacking transparent bi-dimensional cuts [17], projection along one dimension [10], iso-reflectivity surfaces [27], etc.) to render an image resembling those from conventional optical cameras. Thus, the main purpose of the presented system is to estimate the model reflectivity from the microwave data. As a complement, a model point cloud representing the visible surface of the scene under test is also computed. Both representations are continuously updated for each new position of the imager.

1) Flowchart: The global flowchart of the processing algorithm is shown in Fig. 3. This flowchart consists of a continuous loop, which constantly updates the image data. First, a query is sent to two different processes (grouped by dashed lines in Fig. 3), which are implemented and run in a single or multiple different computers. This query triggers the acquisition of the data from the microwave and depth cameras. The trigger is implemented by a simple self-developed protocol based on non-blocking Transmission Control Protocol (TCP) sockets. Subsequently, each data stream is processed independently, as shown in the next sections. At the conclusion of both independent processes, the local reflectivity data and a transformation matrix are available, which can be used to update the target model reflectivity (i.e., microwave image). In addition, the model point cloud is updated.

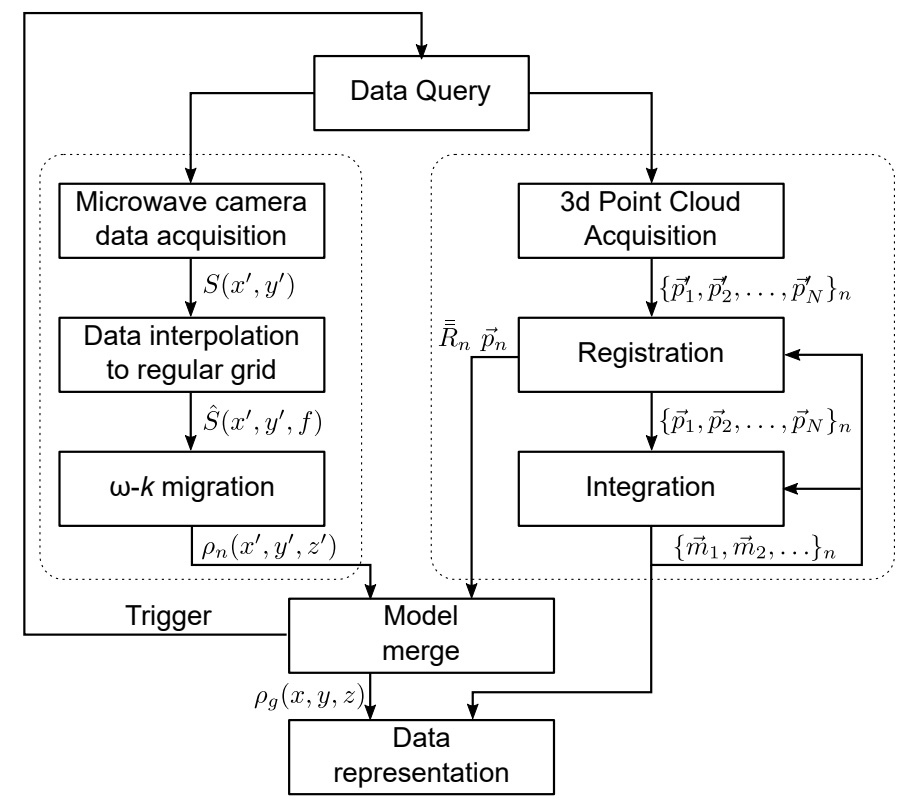

Fig. 3. Flowchart for the multiview portable imager. The areas enclosed by dash lines denote the two parallel process executed at each position: i) local electromagnetic image processing, and ii) position estimation. 
2) Local electromagnetic image processing: For the microwave camera, the first step involves interpolating the data into a regular grid. This step is performed using a linear interpolation based on Sherphard's method [28] implemented by means of a look-up table resulting in a regular lattice of $61 \times 66$ points along the local plane $x^{\prime} y^{\prime}$ with a spacing between them of $\Delta x^{\prime}=2.75 \mathrm{~mm}$ and $\Delta y^{\prime}=2 \mathrm{~mm}$ (see Fig. 4). These numbers are based on the spacing in the physical array. In particular, there is an average non-uniform spacing of $\sim 2 \mathrm{~mm}$ between the receivers along the $y$-axis [17]. Moreover, the spacing between boards in $x$ is $11 \mathrm{~mm}$ and, therefore, its closest divisor to 2 , which is $2.75 \mathrm{~mm}$, is chosen.

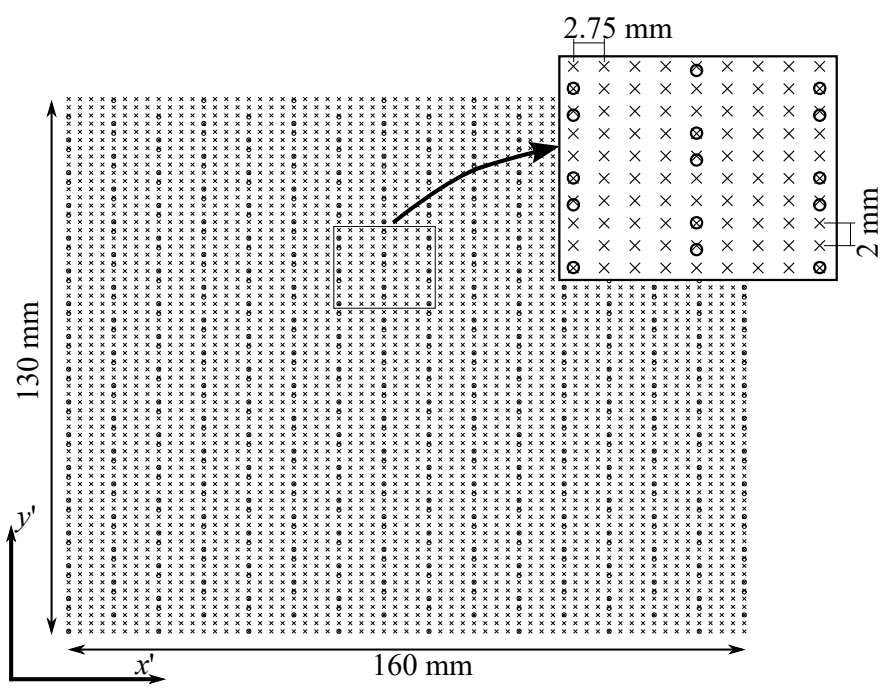

Fig. 4. Local systems of coordinates of the microwave camera: (o) Acquisition points and (x) interpolated points.

The local system of coordinates (denoted by primed coordinates), which moves along with the imager, is chosen so that the central point of the aforementioned regular grid is the origin of coordinates $\left(x^{\prime}, y^{\prime}, z^{\prime}\right)=(0,0,0)$. Fig 5 depicts an example of different local system of coordinates for two different imager positions along with the global system of coordinates.

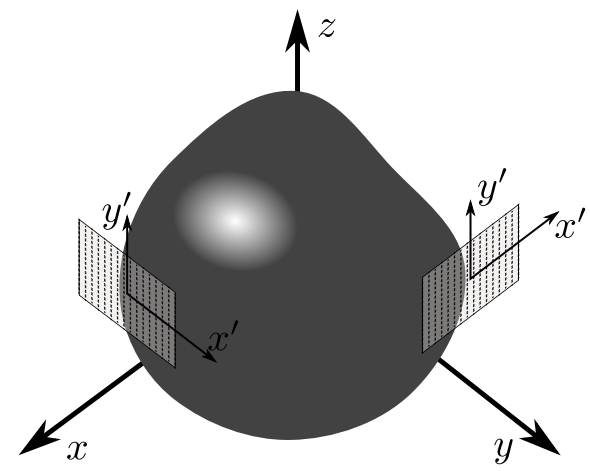

Fig. 5. Global system of coordinates and local system of coordinates for two positions of the imager.

The weights of the look-up table are calculated by performing a Delaunay triangulation of the acquisition points. Next, the three vertices of the triangle surrounding each point of the interpolation grid are found. Finally, three weights, one for each vertex of the triangle, are computed for each point of the interpolation grid. These weights correspond to the area of the three inner triangles normalized by the total area. Thus, the interpolated field $\hat{S}$ at a point $\left(x_{c}^{\prime}, y_{c}^{\prime}\right)$ is given by:

$$
\hat{S}\left(x_{c}^{\prime}, y_{c}^{\prime}, f\right)=\frac{1}{A} \sum_{i=1}^{3} A_{i} S\left(x_{i}^{\prime}, y_{i}^{\prime}, f\right),
$$

where $\left(x_{i}^{\prime}, y_{i}^{\prime}\right)$ are the vertices of the $i$-th triangle, $A_{i}$ is the area of each inner triangle and $A=\sum_{i=1}^{3} A_{i}$ is the area of the overall triangle and $S\left(x_{i}^{\prime}, y_{i}^{\prime}, f\right)$ is the field acquired by the microwave camera. This operation is depicted in Fig. 6.

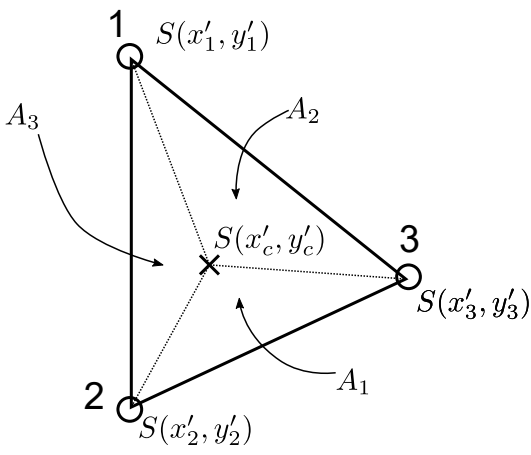

Fig. 6. Interpolation algorithm: (o) denotes acquisition point and (x) denotes interpolation point.

Since the described weights only depend on the acquisition and interpolation points, which are independent of the imager position, they are precomputed together with the look-up table indexes instead of being computed on-the-fly.

Next, a $\omega-k$ migration algorithm is implemented [26], [4] by means of efficient fast Fourier transforms (FFTs) to compute the local reflectivity. Thus, the local reflectivity for the $n$-th scanner position is given by:

$$
\rho_{n}\left(x^{\prime}, y^{\prime}, z^{\prime}\right)=\mathcal{F}^{-1}\left\{\mathcal{F}\left\{\hat{S}_{n}\left(x^{\prime}, y^{\prime}, f\right)\right\} e^{-j k_{z} z_{0}}\right\},
$$

where, $\rho_{n}\left(x^{\prime}, y^{\prime}, z^{\prime}\right)$ is the local reflectivity in local coordinates, $\hat{S}_{n}\left(x^{\prime}, y^{\prime}\right)$ denotes the field evaluated at the interpolation points for the $n$-th imager position, $\mathcal{F}$ denotes Fourier transform and $k_{z}=\sqrt{(2 k)^{2}+k_{x}^{2}+k_{y}^{2}}$ being $k$ the wavenumber and $k_{x}-k_{y}$ the spectral coordinates corresponding to the first Fourier transform. The parameter $z_{0}$ corresponds to the standoff distance along the $z^{\prime}$ axis from the center of the interpolation points to the center of the local reconstruction volume. In practice, the interpolated data are zero-padded to mitigate aliasing artifacts. For this reason, 12 and 20 acquisition points along the $x$ - and $y$-directions, respectively, are added to the interpolated data $\hat{S}\left(x^{\prime}, y^{\prime}\right)$.

3) Position estimation: The depth camera data are acquired and processed in parallel to the operations corresponding to the microwave camera. The core of the depth camera processing is the iterative closest point algorithm (ICP) [29]. This algorithm consists of two main steps: i) registration and ii) integration. The registration step is devoted to finding a transformation so that depth data acquired are aligned with a representation 
of the previously-acquired data. Thus, at the $n$-th imager position, the inputs for the first step (registration) are the last acquired point cloud, denoted by $\left\{\vec{p}_{1}^{\prime}, \vec{p}_{2}, \ldots, \vec{p}_{N}\right\}_{n}$, and the model point cloud, $\left\{\vec{m}_{1}, \vec{m}_{2}, \ldots\right\}_{n}$. On one hand, the last acquired point cloud is a set of $N$ points, which are expressed in local (and, therefore, primed) coordinates. On the other hand, the model point cloud is a set of points from previous acquisitions, expressed in global (non-primed) coordinates, which is calculated during the integration step. Once the registration step is complete, a rotation matrix $\bar{R}_{n}$ and a vector $\overrightarrow{t_{n}}$ are available, and consequently, the imager position and attitude can be determined. Subsequent to these steps, the integration step updates the model point cloud by merging it with the last acquired point cloud. The criterion to include a certain point into the model point cloud is based on maximum distance and angle criteria, as described in [20]. It is important to note that this point cloud, in contrast to the point clouds acquired by the depth camera, does not have a fixed number of points as it is updated for each imager position.

Since we aim to obtain imaging results in real or quasireal time for many applications, such as those related to security screening, the ICP code must be optimized to achieve an execution time compatible with being real-time. Thus, in order to speed up the run time, a parallelized version of our previous offline ICP-based positioning system [20], adapted from the Point Cloud Library (PCL) [30], was implemented and customized to operate in real-time.

Taking into account that the developed tool is intended to be used in conjunction with small shared-memory systems (i.e., personal computers (PC) or laptops with multicore CPUs), the authors have incorporated a parallelization scheme based on OpenMP [31] for the registration and integration steps. Concerning the registration step, the calculation of the transformation matrix for the Point Cloud (PC) obtained with each view has been parallelized, so that several points in the PC can be processed simultaneously. The code related to the integration that has been parallelized deals with the generation of new vertices, in order to accelerate the merging of each registered PC with the growing model mesh.

It is important to note that, in addition to the transformation, the model point cloud is also available during the execution of the algorithm. These data can be useful in some situations as a complement to the electromagnetic model calculated from the microwave camera data. For example, for people screening applications, it can be useful to check whether an apparent protrusion is due to a concealed object or to a clothing crease/clump.

4) Data merge: At the end of the local electromagnetic image computation and the position estimation, the model reflectivity, denoted by $\rho_{n}^{(g)}(x, y, z)$, can be updated with the last computed local reflectivity. For this purpose, the local reflectivity is first translated into the global system of coordinates with the help of the rotation matrix, $\overline{\bar{R}}_{n}$, and position vector $\vec{t}_{n}$. According to the study carried out in [20], the positioning error provided by this positioning system is expected to be in the order of several millimeters, and consequently, is close to the operational microwave wavelength. As previously discussed, the optimal choice in these cases is to implement a non-coherent merging formulation. In particular, the formulation presented in [18] is used as it enables to merge multiple overlapping local reflectivities while preserving the maximum reflectivity values, as they are usually associated to areas of interest. Thus, the model reflectivity is updated as:

$$
\rho_{n}^{(g)}(x, y, z)=\max \left\{\rho_{n-1}^{(g)}(x, y, z), \rho_{n}(x, y, z)\right\} .
$$

Finally, the model reflectivity and point cloud can be visually illustrated providing insightful information about the model about the inner and outer layers of the object under test, respectively.

\section{RESULTS}

The results presented here are focused on security applications. All of these results have been conducted by performing different arbitrary sweeps along a mannequin wearing a shirt with different concealed objects hidden by the shirt. In order to get a reflection closer to the human skin reflectivity, the plastic mannequin is covered by conductive paint [32], [33], which has a sheet resistance of $32-55 \Omega /$ sq [34]. In practice, the human body reflectivity is expected to exhibit some differences from the target (i.e., handgun, knife, etc.) reflectivity, increasing the contrast of the image.

The resolution of the depth camera was set to $640 \times 240$. Moreover, a clipping box of $10 \times 10 \times 20 \mathrm{~cm}^{3}$ is used to reduce the number of points in the acquired point clouds reducing the computational time for estimating the position. The rest of the relevant ICP algorithm parameters are summarized in Table I. In this table, $\epsilon_{d}$ is the difference of the average squared distance between the (transformed) last acquired point cloud and the correspondences in the model point cloud. Additional description of the remaining parameters is given in [30]. The local electromagnetic image processing, merging of the data and data representation were performed using an Intel® $2.4 \mathrm{GHz}$ Xeon ${ }^{\circledR}$ E5645 with $36 \mathrm{~GB}$ of RAM. The position estimation was performed in an Intel® $2.4 \mathrm{GHz}$ Core(TM) i74700MQ laptop with $16 \mathrm{~GB}$ of RAM.

Table I

MOST RELEVANT ICP PARAMETERS USED IN THE PRESENTED EXPERIMENTS.

\begin{tabular}{|c|c|c|}
\hline \multirow{3}{*}{ Registration } & $\epsilon_{d}$ & $4.5 \cdot 10^{-5} \mathrm{~cm}^{2}$ \\
\cline { 2 - 3 } & Max. Iterations & 12 \\
\cline { 2 - 3 } Integration & Min. Overlap & $55 \%$ \\
\hline \multirow{3}{*}{} & Maximum age & 15 \\
\cline { 2 - 3 } & Maximum angle & $10^{\circ}$ \\
\cline { 2 - 3 } & Visibility & 2 \\
\hline
\end{tabular}

The imager is depicted in Fig. 7. The depth and microwave cameras were carefully aligned to reduce the number of transformations. Nevertheless, due to physical constraints, an offset between both cameras along the local coordinates $y^{\prime}$ and $z^{\prime}$ of $185 \mathrm{~mm}$ and $-70 \mathrm{~mm}$, respectively, was unavoidable. Although these offsets are easily corrected in post-processing, the vertical offset entails that the areas imaged by the depth and microwave cameras do not fully overlap. It is interesting to note that other promising transmitting and receiving layouts, such as the ones based on sparse multi-static arrays [19], 
exhibit a larger gap between the elements, where the depth camera can be positioned, overcoming this issue.

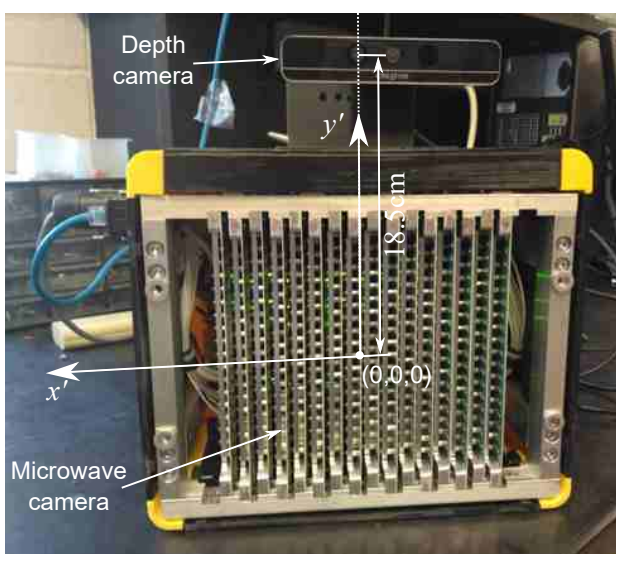

Fig. 7. Imaging system (i.e., imager) comprising depth and microwave cameras. The local system of coordinates and the vertical offset are also shown.

In the first example, the mannequin is placed on a turntable, which is manually rotated. The microwave camera with the attached depth camera is oriented so that its normal vector is pointing towards the axis of the turntable. Thus, it emulates the circular movement of the microwave camera around the mannequin. This turntable setup is chosen to constrain the movement and check whether the positions were correctly estimated. In this example, a protrusion was generated by attaching a metallic bar to the mannequin's body, as shown in Fig. 8.

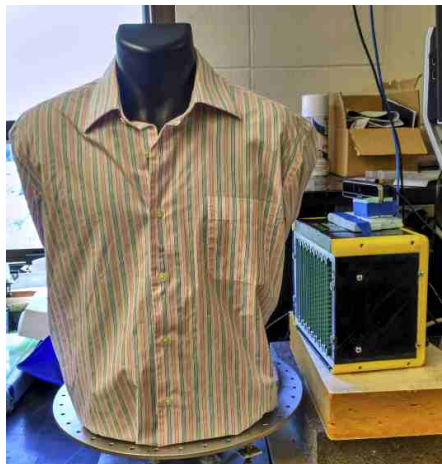

(a)

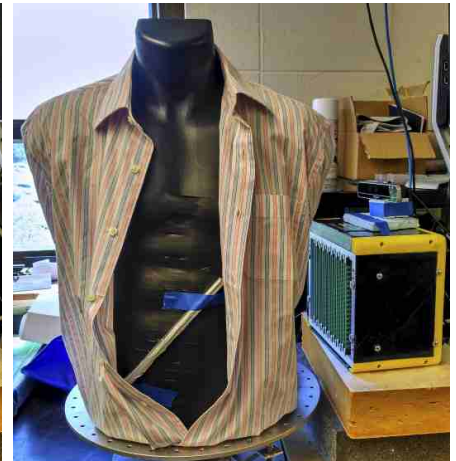

(b)
Fig. 8. Turntable and mannequin with a protrusion created by an aluminum foil bar.

The turntable is moved so that it covers an arc of $100^{\circ}$ with the distance between the microwave camera aperture and the rotation axis being $d=29.0 \mathrm{~cm}$. Within this movement (rotation), the data was automatically acquired at 44 locations. The employed imaging domain and the estimated positions are shown in Fig. 9. In addition, the normal vectors to the $x^{\prime} y^{\prime}$ planes are also shown. As it is clearly seen, all of these normal vectors are approximately crossing at a distance equal to the radius of the described arc $(29 \mathrm{~cm})$. The total arc estimated by the positioning system is $87.9^{\circ}$ instead of the actual $100^{\circ}$, i.e., the cumulative angle error along the trajectory of this example is $-12.1 \%$.
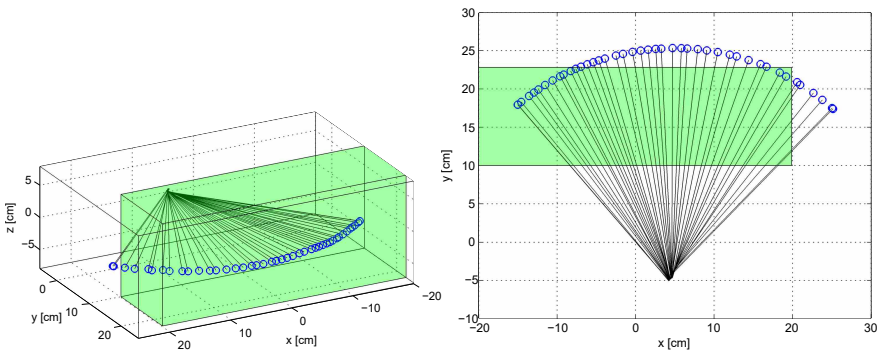

Fig. 9. Imaging domain and estimated positions for the mannequin with a protrusion: (a) oblique and (b) top views.

In order to evaluate the impact of the positioning errors on the microwave image, several intermediate images are depicted in Fig. 10. In this figure, the color scheme corresponds to the position of the reflectivity maxima along the $y$-axis weighted by the reflectivity value, as described in [20]. These results show that to produce a viable image one needs to obtain microwave data from a sufficiently high number of positions. The most visible artifact in these images (b-d) is a slight bend at the top portion of the bar due to the cumulative error in the roll angle estimation. Although this kind of local artifacts are common when scanning with depth cameras [35], they do not prevent the target from being correctly identified.

In the next example, the same setup is considered but the metallic bar is replaced by a knife with a plastic handle, as shown in Fig. 11(b). The same distance from the camera to the turntable was considered as well. However, in this case the arc length was $95^{\circ}$.

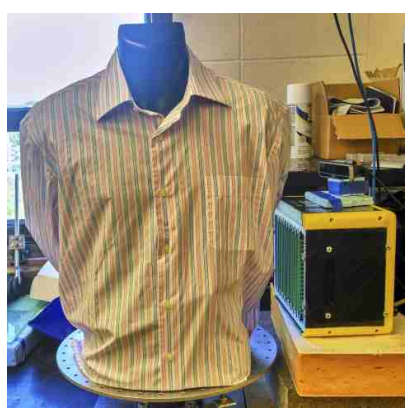

(a)

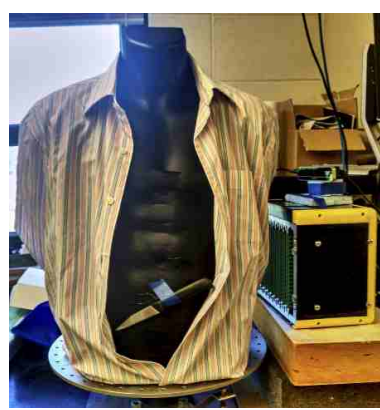

(b)
Fig. 11. Turntable and mannequin with a concealed knife.

The imaging domain and estimated positions for this case are shown in Fig. 12. In this case, a total of 43 acquisitions were performed along the total arc. The positioning system estimated that the movement was performed along $84.4^{\circ}$, i.e., the total cumulative error for this angle is $-11.17 \%$. 


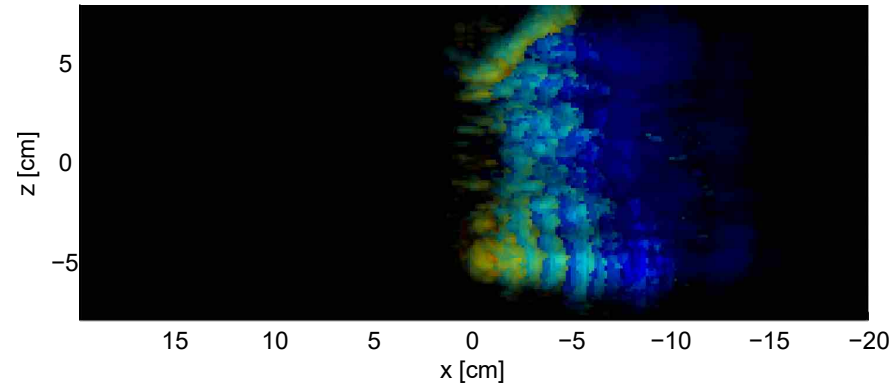

(a)

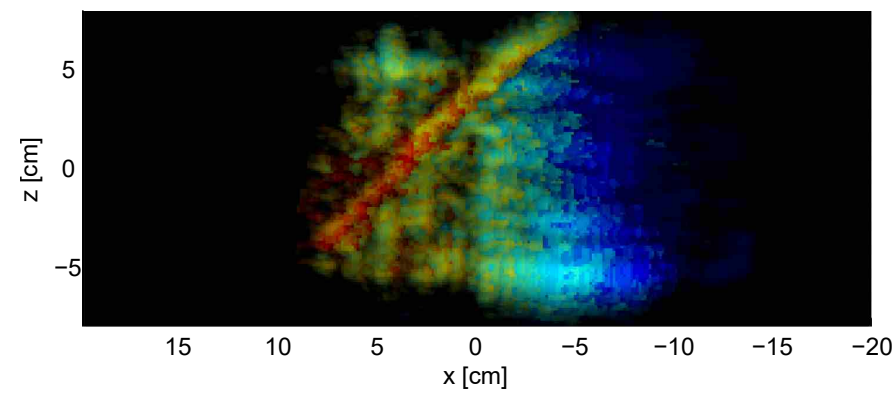

(b)

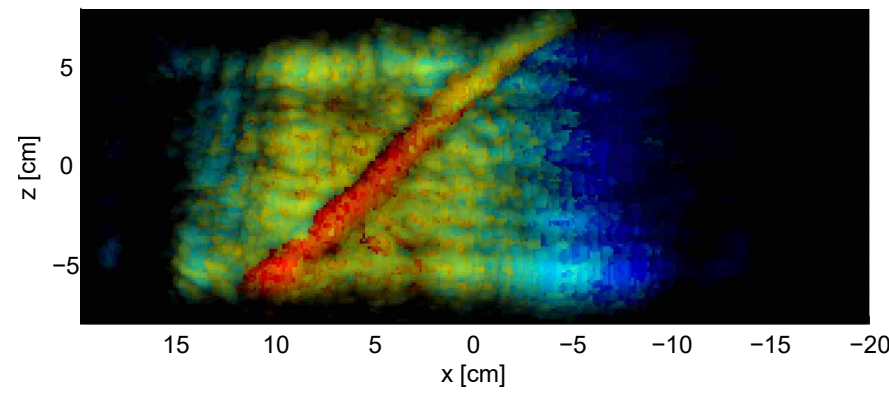

(c)

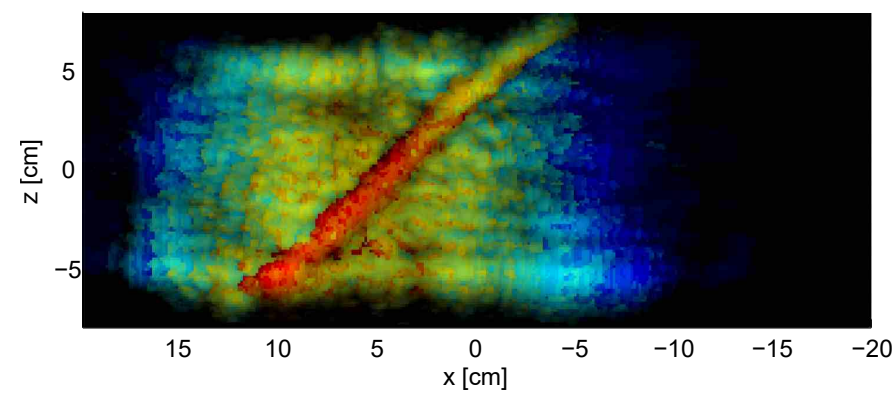

(d)

Fig. 10. Image composed from model reflectivity for the metallic bar attached example using (a) 11 positions, (b) 22 positions, (c) 33 positions and (d) 44 positions.

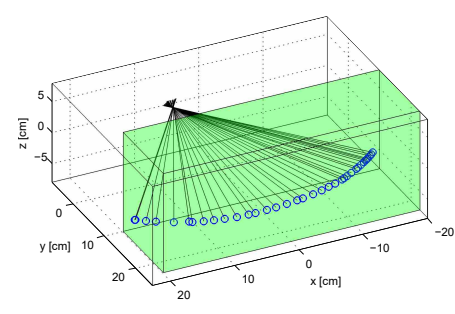

(a)

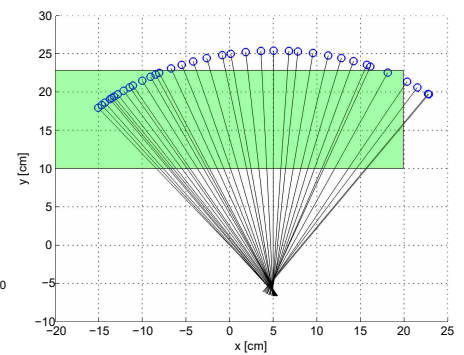

(b)
Fig. 12. Imaging domain and estimated positions for the mannequin with an attached knife: (a) oblique and (b) top views.
Several intermediate images corresponding to this case are shown in Fig. 13. In this case, the metallic blade is clearly seen whereas a shadow around the plastic handle with the tang of the knife is also visible in Fig. 13(d).

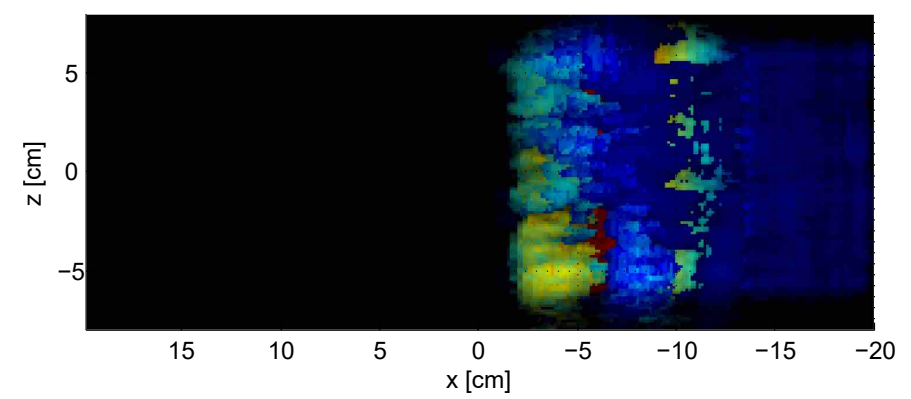

(a)

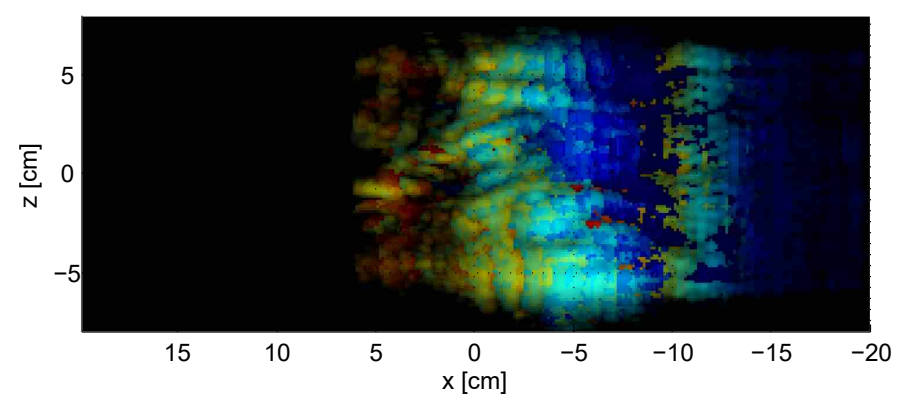

(b)

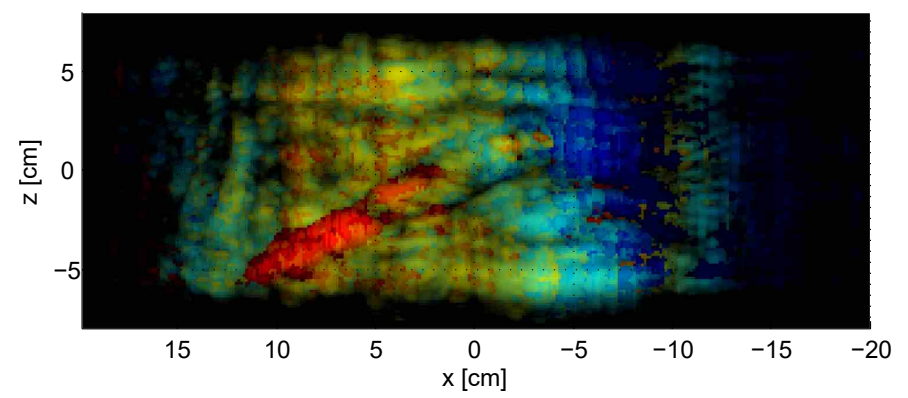

(c)

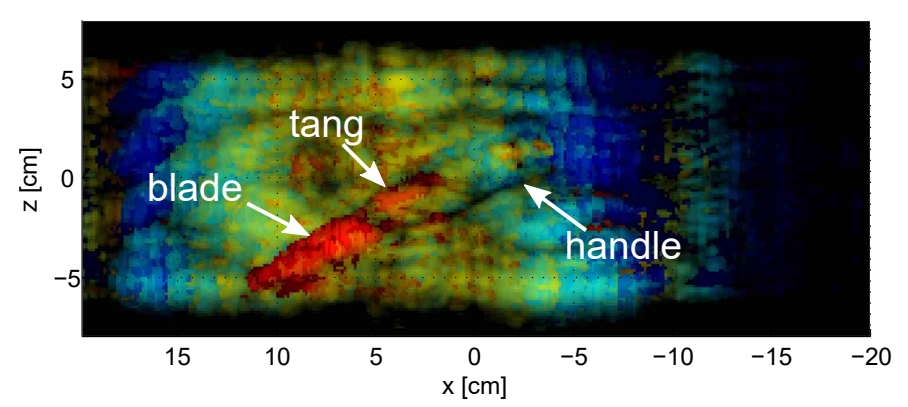

(d)

Fig. 13. Image composed from model reflectivity for the attached knife example using: (a) 10 positions, (b) 20 positions, (c) 30 positions and (d) 43 positions.

In the third case, an arbitrary movement, where the imager is moved by hand without using the turntable, is considered in which the mannequin has a BB handgun attached to his back, as shown in Fig. 14. The BB handgun, which is made of plastic, is also covered by conductive paint for this experiment. 


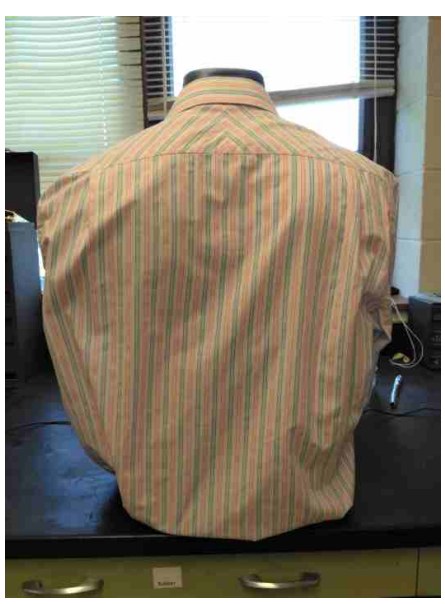

(a)

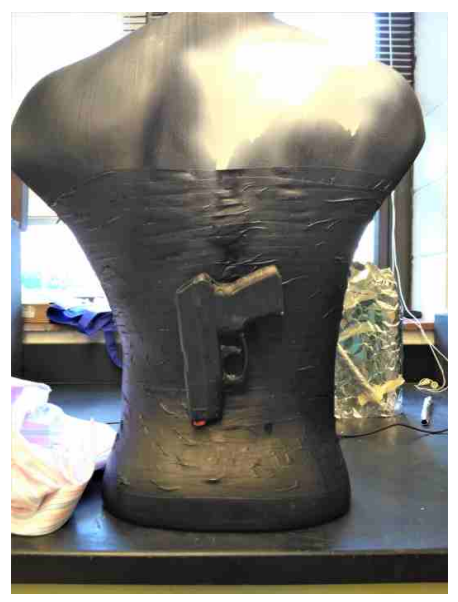

(b)

Fig. 14. Mannequin with an attached BB handgun.

The imaging domain and estimated trajectory during this scan together with the $z^{\prime}$ axis at each position are shown in Fig. 15. The reconstructed electromagnetic image is depicted in Fig 16 revealing that the gun can be clearly detected by this proposed system and approach. The computation times for this fully arbitrary scan are summarized in Table II.

Table II

COMPUTATIONAL TIMES FOR THE PROCESSING OF THE DATA AT EACH POSITION IN THE EXAMPLE OF A MANNEQUIN WITH AN ATTACHED GUN.

\begin{tabular}{cccc} 
& Min. [ms] & Max. [ms] & Avg. [ms] \\
\hline Position estimation & 1294 & 1485 & 1379 \\
\hline Local EM image & 578 & 761 & 643 \\
\hline Data merge and representation & 328 & 349 & 335 \\
\hline Total time & 1627 & 1820 & 1715 \\
\hline
\end{tabular}

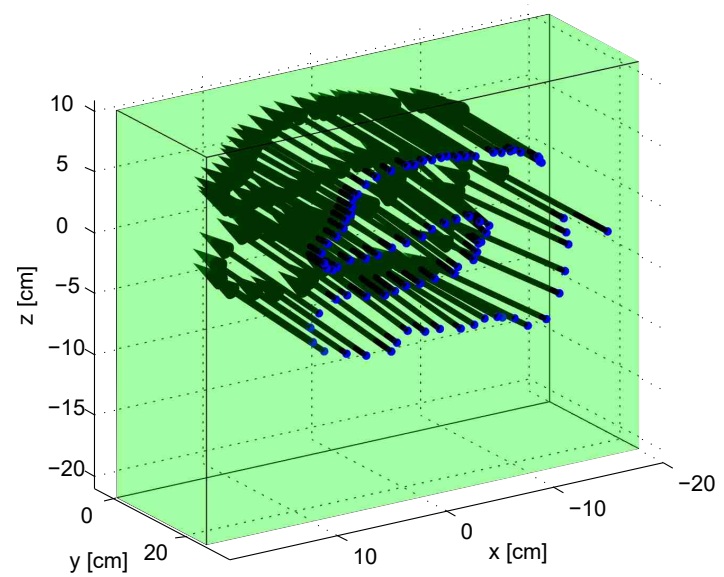

Fig. 15. Trajectory and reconstruction volume for the mannequin with attached BB gun.

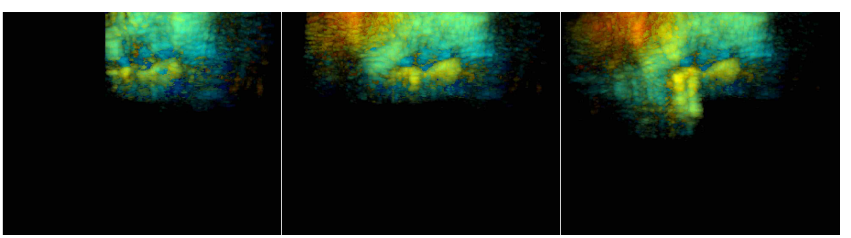

(a)

(b)

(c)

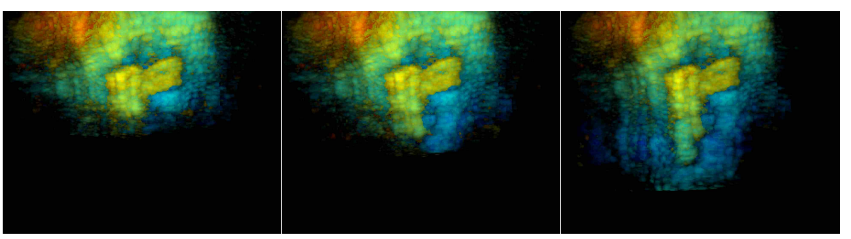

(d)

(e)

(f)

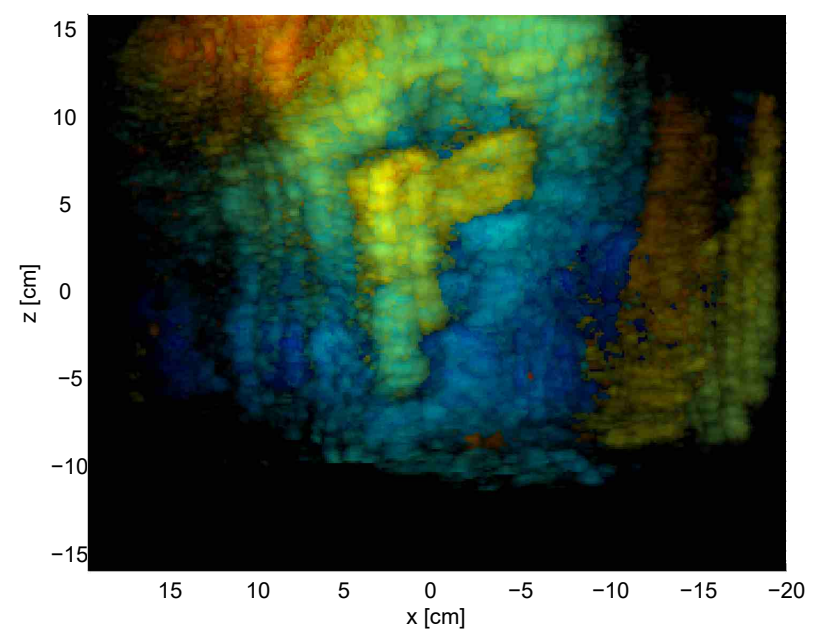

(g)

Fig. 16. Image composed from model reflectivity for the BB handgun example using: (a) 12 positions, (b) 24 positions, (c) 36 positions, (d) 48 positions, (e) 60 positions, (f) 72 positions and (g) 86 positions.

Fig. 17 shows an overlay of the model reflectivity and point cloud for this case. It is important to note that there is a clear offset between the area imaged by the depth camera and the area imaged by the microwave camera, as a result of the aforementioned vertical offset of $185 \mathrm{~mm}$.

\section{CONCLUSIONS}

The first demonstrator of a real-time multi-view imager has been presented. The use of a monostatic, portable, highresolution, 3D camera for the microwave data acquisition and a depth camera for positioning systems results in a handheld (portable) imaging systems, which can be arbitrarily moved around the object being imaged. The imager described here provided a continuously-updated image with a refresh rate of approximately 1.5 seconds. Although it has been shown in the literature that the microwave data can be processed in realtime at a relatively high video frame-rates (e.g., $\sim 30 \mathrm{fps}$ for the microwave camera utilized here [17]) with the help of GPUs, the current bottleneck is due to the time required to estimate the position from the depth camera data and, therefore, future research should focus on this point. Despite of that, the system showed to be quite responsive and enables sufficient resolution 


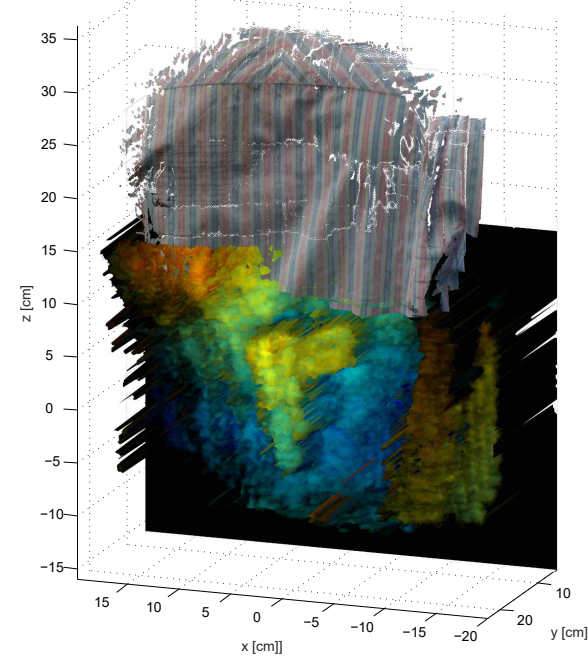

Fig. 17. Overlay of the 3D electromagnetic image and model point cloud for the BB gun example.

to easily identify objects of interest for security and contraband detection applications.

The presented imager paves the way towards the development of free-hand scanners with capabilities to image large areas by simple and arbitrary movements. In the area of security screening, this type of imager opens a way to avoid booth scanner devices and it enables the possibility of scanning local areas mitigating privacy concerns, while providing a relatively high screening throughput rate. Such a system can also be deployed on demand as complimentary to booth scanners to increase screening throughput or to areas such as sporting and other public events where permanently deployed (i.e., booth) systems are not practical. Moreover, the portable imager can be intuitively guided with the help of dynamic images so that after finding something suspicious (e.g., a part of a protrusion) the surroundings can be easily inspected further.

Future work includes, in addition to speeding up the positioning system, experimentation with non-static targets. Even in the case of collaborative beings, a small movement is expected due to natural movements (e.g., respiration). Since each local images are taken in $\sim 30 \mathrm{~ms}$, the results are not expected to be adversely affected by these movements. However, the global images composed by merging local images are expected to contain moderate artifacts due to a relatively rapid motion. Since a moderate (involuntary) movement is expected to be in the order of a few millimeters, merging different images of the same area any defocusing effect is expected to be in the same order as that of the positioning error, as described earlier.

In addition, although the merge algorithm has exhibited a robust behavior in the experimentation, it would be useful, in the future works, to include the possibility of re-imaging some areas in case a clear error or an artifact is observed.

\section{REFERENCES}

[1] M. Pastorino, Microwave Imaging. John Wiley \& Sons, Inc., 2010.
[2] S. Kharkovsky and R. Zoughi, "Microwave and millimeter wave nondestructive testing and evaluation - overview and recent advances," IEEE Instrumentation Measurement Magazine, vol. 10, no. 2, pp. 26-38, April 2007.

[3] M. T. Ghasr, D. Pommerenke, J. T. Case, A. McClanahan, A. AflakiBeni, M. Abou-Khousa, S. Kharkovsky, K. Guinn, F. D. Paulis, and R. Zoughi, "Rapid rotary scanner and portable coherent wideband Qband transceiver for high-resolution millimeter-wave imaging applications," IEEE Transactions on Instrumentation and Measurement, vol. 60, no. 1, pp. 186-197, Jan 2011.

[4] D. M. Sheen, D. L. McMakin, and T. E. Hall, "Three-dimensional millimeter-wave imaging for concealed weapon detection," IEEE Trans. Microw. Theory Tech., vol. 49, no. 9, pp. 1581-1592, September 2001.

[5] N. K. Nikolova, "Microwave imaging for breast cancer," IEEE Microwave Magazine, vol. 12, no. 7, pp. 78-94, Dec 2011.

[6] A. T. Mobashsher and A. M. Abbosh, "On-site rapid diagnosis of intracranial hematoma using portable multi-slice microwave imaging system," Sci. Rep, vol. 6, no. 37620, 2016.

[7] J. Case, M. Ghasr, and R. Zoughi, "Optimum 2-D non-uniform spatial sampling for microwave SAR-based NDE imaging systems," IEEE Transactions on Instrumentation and Measurement, vol. 61, no. 11, pp. 3072-3083, Nov 2012.

[8] M. Ghasr, S. Kharkovsky, R. Bohnert, B. Hirst, and R. Zoughi, "30 $\mathrm{GHz}$ linear high-resolution and rapid millimeter wave imaging system for NDE," IEEE Transactions on Antennas and Propagation, vol. 61, no. 9, pp. 4733-4740, Sep 2013.

[9] M. Fallahpour, J. Case, M. Ghasr, and R. Zoughi, "Piecewise and wiener filter-based SAR techniques for monostatic microwave imaging of layered structures," IEEE Transactions on Antennas and Propagation, vol. 62, no. 1, pp. 282-294, Jan 2014

[10] S. Ahmed, A. Schiessl, and L. Schmidt, "A novel fully electronic active real-time imager based on a planar multistatic sparse array," IEEE Trans. Microw. Theory Tech., vol. 59, no. 12, pp. 3567-3576, 2011.

[11] F. Gumbmann and S. S. Ahmed, "Walk through screening with multistatic mmW technology," in Proc. SPIE 9993, Millimetre Wave and Terahertz Sensors and Technology IX, 2016, p. 999306; DOI: $10.1117 / 12.2247183$.

[12] — , "Novel walk-through imaging system for security screening," SPIE Newsroom, p. ; DOI: 10.1117/2.1201609.006703, 2016.

[13] B. Gonzalez-Valdes, Y. Alvarez, Y. Rodriguez-Vaqueiro, A. ArboleyaArboleya, A. Garcia-Pino, C. M. Rappaport, F. Las-Heras, and J. A. Martinez-Lorenzo, "Millimeter wave imaging architecture for on-themove whole body imaging," IEEE Trans. Antennas Propag., vol. 64, no. 6, pp. 2328-2338, Jun 2016.

[14] J. Laviada, Y. Álvarez, A. Arboleya, and B. G.-V. F. Las-Heras, "Multiview techniques for mm-wave imaging," in IEEE AP-S Symposium on Antennas and Propagation, San Diego, USA, July 9-14 2017.

[15] M. Pollefeys, L. Gool, M. Vergauwen, F. Verbiest, K. Cornelis, J. Tops, and R. Koch, "Visual modeling with a hand-held camera," International Journal of Computer Vision, vol. 59, no. 3, pp. 207-232, 2004.

[16] P. Tanskanen, K. Kolev, L. Meier, F. Camposeco, O. Saurer, and M. Pollefeys, "Live metric 3D reconstruction on mobile phones," in International Conference on Computer Vision (ICCV 2013), Sidney, Australia, 3-6 Dec 2013.

[17] M. T. Ghasr, M. J. Horst, M. R. Dvorsky, and R. Zoughi, "Wideband microwave camera for real-time 3-d imaging," IEEE Transactions on Antennas and Propagation, vol. 65, pp. 258 - 268, Jan 2017.

[18] J. Laviada, A. Arboleya, Y. Álvarez López, B. González-Valdés, and F. Las-Heras, "Multiview three-dimensional reconstruction by millimetre-wave portable camera," Scientific Reports, vol. 7, 2017, doi:10.1038/s41598-017-06475-7.

[19] J. Laviada, A. Arboleya, and F. Las-Heras, "Multistatic millimeter-wave imaging by multiview portable camera," IEEE Access, vol. 5, pp. 19259 - 19268, 2017.

[20] J. Laviada, M. López-Portugués, A. Arboleya, and F. Las-Heras, "Multiview mm-wave imaging with augmented depth camera information," IEEE Access, pp. 2169-3536, 2018.

[21] J. Laviada, M. T. Ghasr, F. Las-Heras, and R. Zoughi, "Multiview imaging with real-time microwave camera from known positions," in IEEE AP-S Symposium on Antennas and Propagation, Boston, USA, July 8-13 2018

[22] R. Hartley and A. Zisserman, Multiple View Geometry in Computer Vision. Cambridge University Press, 2003.

[23] M. T. Ghasr, M. A. Abou-Khousa, S. Kharkovsky, R. Zoughi, and D. Pommerenke, "Portable real-time microwave camera at 24 ghz," IEEE Transactions on Antennas and Propagation, vol. 60, pp. 1114-1125, Feb 2012. 
[24] Intel Corporation. (2017) Intel® RealSense ${ }^{\mathrm{TM}}$ Camera SR300. A depthsensing camera that uses coded-light methodology for close-range depth perception. [Online]. Available: https://software.intel.com/enus/realsense/sr300

[25] R. A. Morano, C. Ozturk, R. Conn, S. Dubin, S. Zietz, and J. Nissano, "Structured light using pseudorandom codes," IEEE Transactions on Pattern Analysis and Machine Intelligence, vol. 20, no. 3, pp. 322-327, Mar 1998.

[26] J. M. Lopez-Sanchez and J. Fortuny-Guasch, "3-D radar imaging using range migration techniques," IEEE Trans. Antennas Propag., vol. 48, no. 5, pp. 728-737, May 2000.

[27] J. Laviada and F. Las-Heras, "Scalar calibration for broadband synthetic aperture radar operating with amplitude-only data," IEEE Antennas and Wireless Propagation Letters, vol. 14, pp. 1714-1717, 2015.

[28] D. Shepard, "A two-dimensional interpolation function for irregularlyspaced data," in Proceedings of the 1968 ACM National Conference, 1968, pp. 517-524.

[29] P. J. Besl and N. D. McKay, "A method for registration of 3-D shapes," IEEE Transactions on Pattern Analysis and Machine Intelligence, vol. 14, no. 2, pp. 239-256, Feb 1992.

[30] R. B. Rusu and S. Cousins, "3D is here: Point Cloud Library (PCL)," in IEEE International Conference on Robotics and Automation (ICRA), Shanghai, China, May 9-13 2011.

[31] The OpenMP ARB. (2016) The OpenMP API specification for parallel programming. [Online]. Available: http://www.openmp.org/

[32] D. L. McMakin, D. M. Sheen, T. E. Hall, M. O. Kennedy, and H. P. Foote, "Biometric identification using holographic radar imaging techniques,” vol. 6538, Orlando, United States, 2007, pp. 6538 - 6538 -12 .

[33] J. N. Gollub, O. Yurduseven, K. P. Trofatter, D. Arnitz, M. F. Imani, T. Sleasman, M. Boyarsky, A. Rose, A. Pedross-Engel, H. Odabasi, T. Zvolensky, G. Lipworth, D. Brady, D. L. Marks, M. S. Reynolds, and D. R. Smith, "Large metasurface aperture for millimeter wave computational imaging at the human-scale," Scientific Reports, vol. 7, no. 42650, Feb 2017.

[34] http : / / www . bareconductive . com / wp - content / uploads / 2016 / 05 / ElectricPaint_ApplicationNotes.pdf, Accessed: August 2, 2018.

[35] Y. Cui, S. Schuon, S. Thrun, D. Stricker, and C. Theobalt, "Algorithms for 3d shape scanning with a depth camera," IEEE Transactions on Pattern Analysis and Machine Intelligence, vol. 35, no. 5, pp. 1039 1050, May 2013. 